\title{
Ion chemistry in the interstellar medium
}

\author{
Oscar Martinez Jr., Theodore P. Snow, and Veronica M. Bierbaum \\ Department of Chemistry and Biochemistry, Center for Astrophysics and Space Astronomy, \\ Department of Astrophysical and Planetary Sciences, University of Colorado, \\ Boulder, CO 80309-0215, U.S.A. \\ email: oscar.martinez@colorado.edu, theodore.snow@colorado.edu, \\ veronica.bierbaum@colorado.edu
}

\begin{abstract}
Without accurate data on reaction rates and branching ratios, models of interstellar chemistry are unreliable. Recent research has identified a number of reactions of unusual importance because the rates and branching ratios are unknown or poorly known. Efforts to expand and improve on current databases are underway using a flowing afterglow-selected ion flow tube (FA-SIFT) coupled to a quadrupole mass spectrometer. Our current focus is on the reactions of $\mathrm{C}^{+}$, a major cation in the interstellar medium, with the neutrals $\mathrm{O}_{2}, \mathrm{H}_{2} \mathrm{O}, \mathrm{CH}_{4}, \mathrm{NH}_{3}$ and $\mathrm{C}_{2} \mathrm{H}_{2}$. Future planned work includes studies of polycyclic aromatic hydrocarbons (PAHs), developing comprehensive pathways for their formation, and identification of those PAHs important to interstellar chemistry. The recent discovery of ISM anions has highlighted the importance of examining mechanisms of anionic chemistry in the interstellar medium, and we plan to obtain data relevant to the formation and destruction processes of molecular anions in space.
\end{abstract}

Keywords. Astrobiology, astrochemistry, astronomical data bases: miscellaneous, ISM: evolution, ISM: kinematics and dynamics, ISM: molecules

\section{Introduction}

There exist numerous discrepancies in rate constants and branching ratios compiled into chemical databases available to the astrophysical community. A study by MarkwickKemper (2005) ranked the importance of reactions used in astrochemical models by examining the effect of uncertainties in rate constants and branching ratios. Of the $\sim 4000$ reactions in the UMIST 99 catalog, only 1200 reactions have less than $25 \%$ uncertainty and $\sim 2700$ reactions have certainty only "within a factor of 2 ".

Of particular importance to the evolution and chemistry of the interstellar medium (ISM) are reactions involving the carbon cation, $\mathrm{C}^{+}$. Found throughout the ISM, its chemistry with interstellar neutrals is prevalent in dense molecular clouds. Unlike diffuse clouds, where carbon is photoionized, in dense molecular clouds $\mathrm{C}^{+}$is formed via the reaction $\mathrm{He}^{+}+\mathrm{CO} \longrightarrow \mathrm{C}^{+}+\mathrm{O}+\mathrm{He}$ with supplemental ionization by cosmic rays (Herbst \& Klemperer 1973, Watson 1974). $\mathrm{C}^{+}$reactions with $\mathrm{NH}_{3}, \mathrm{CH}_{4}, \mathrm{O}_{2}, \mathrm{H}_{2} \mathrm{O}$ and $\mathrm{C}_{2} \mathrm{H}_{2}$ were among the top 100 in Markwick-Kemper's study; although prior measurements for the reactions have been made, our focus was to eliminate the large uncertainty associated with the prior measurements and to clear the discrepancies between values reported in available databases.

\section{Experimental}

The flowing afterglow selected ion flow tube (FA-SIFT) is ideal for studies of ionneutral reactions relevant to the interstellar medium. A variety of ionization methods and chemical versatility exist for the FA-SIFT method. The FA-SIFT, with its high ion 
Table 1. Recommended reaction rate constants and branching ratio values for reactions of $\mathrm{C}^{+}$ with $\mathrm{NH}_{3}, \mathrm{CH}_{4}, \mathrm{O}_{2}, \mathrm{H}_{2} \mathrm{O}$ and $\mathrm{C}_{2} \mathrm{H}_{2}$.

\begin{tabular}{c|c|c|c}
\hline Reaction & $k\left(\times 10^{-9} \mathbf{c m}^{3} \mathbf{s}^{-1}\right)$ & Products & Branching Ratios \\
\hline $\mathrm{C}^{+}+\mathrm{NH}_{3}$ & $2.36 \pm 0.15$ & $\mathrm{H}_{3} \mathrm{~N}^{+}+\mathrm{C}$ & $28 \pm 12$ \\
& & $\mathrm{CH}_{2} \mathrm{~N}^{+}$ & $67 \pm 11$ \\
& & $\mathrm{CHN}^{+}+\mathrm{H}_{2}$ & $5 \pm 2$ \\
\hline $\mathrm{C}^{+}+\mathrm{CH}_{4}$ & $1.39 \pm 0.054$ & $\mathrm{C}_{2} \mathrm{H}_{2}^{+}+\mathrm{H}_{2}$ & $28 \pm 2$ \\
& & $\mathrm{C}_{2} \mathrm{H}_{3}^{+}+\mathrm{H}$ & $72 \pm 2$ \\
\hline $\mathrm{C}^{+}+\mathrm{O}_{2}$ & $0.796 \pm 0.024$ & $\mathrm{O}^{+}+\mathrm{CO}$ & $57 \pm 12$ \\
& & $\mathrm{CO}^{+}+\mathrm{O}$ & $43 \pm 12$ \\
\hline $\mathrm{C}^{+}+\mathrm{H}_{2} \mathrm{O}$ & $2.09 \pm 0.17$ & $\mathrm{HOC}^{+}+\mathrm{H}$ & N/A \\
\hline $\mathrm{C}^{+}+\mathrm{C}_{2} \mathrm{H}_{2}$ & $1.78 \pm 0.15$ & $\mathrm{C}_{3} \mathrm{H}^{+}+\mathrm{H}$ & N/A \\
\hline
\end{tabular}

densities and sensitivity, is capable of kinetic analysis and product ion characterization (For description in greater detail, see Bierbaum 2003).

Refinement of the $\mathrm{C}^{+}$reactions was made through several means. Experiments were performed on two separate FA-SIFT instruments, each with optimized and calibrated experimental parameters [e.g. reaction flow tube pressure, reaction distance (equivalent to time), carrier gas flow, and neutral reactant flow]. Additionally, the use of lower energy $(\sim 30 \mathrm{eV})$ electron impact ionization maximized ground state $\left({ }^{2} \mathrm{P}\right) \mathrm{C}^{+}$and minimized the more reactive metastable $\left({ }^{4} \mathrm{P}\right) \mathrm{C}^{+}$formation. Lastly, effort was made to eliminate mass discrimination, the effects of which skew product branching ratios.

\section{Results and Discussion}

Excellent agreement was found between the sets of measurements made on both instruments. Precise and accurate evaluation of rate constants and branching ratios, shown in Table 1, are recommended for use by the astrophysical community in modeling the chemistry and physics of the ISM among other pertinent applications. The associated instrumental error reported here is $15 \%$, while the precision coupled to measurements of each reported value is reported as a standard deviation from the value. Rate constants lower than literature values are attributed to the absence of metastable $\mathrm{C}^{+}$. A lower reported reaction rate with $\mathrm{C}_{2} \mathrm{H}_{2}$ has also been attributed to purification of the gas to remove acetone, an additive that prevents polymerization, but is highly reactive with $\mathrm{C}^{+}$.

\section{Acknowledgements}

Kind thanks go out to NASA and its GSRP program for support and to Dr. Farid Salama at the NASA Ames Research Center for his mentorship.

\section{References}

Bierbaum, V. M. 2003, in: Armentrout, P. B. (ed.), The Encyclopedia of Mass Spectrometry. vol.1 Theory and Ion Chemistry (Amsterdam:Elsevier), p. 17

Herbst, E. \& Klemperer, W. 1973, ApJ, 185, 505

Markwick-Kemper, A. J. 2005, in: Lis, D. C., Blake, G. A., \& Herbst, E. (eds.), The most important reactions in gas-phase astrochemical models, Proc. IAU Symposium No. 231

Watson, W. D. 1974, ApJ, 188, 35 